\title{
ABSTRACTS.
}

\section{THEORY OF THE AIRSCREW.}

Professor Georges de Bothezat has contributed to "Aviation" an introductory article on the theory of the airscrew which defines the terms and hypotheses used in his conception of the phenomena associated with screw propulsion. In it the author, late of the Polytechnic Institute, Petrograd, gives the outlines of a new theory of the airscrew.

The term "Relative pitch" is adopted as a fundamental parameter and defined as the pitch of an element of the screw b'ade, measured by taking the pitch of the blade element as unity. The "Specific function" is the relation of the work of the thrust to the work of the torque applied to the screw axis.

An illustrative diagram gives the general analytical condition of all states of work for a typical screw. In this a curve is drawn for the values of "Relativepitch" as abscissæ against the "Specific function" as ordinates. The portion of the curve between the absciss $x=0$ and $x=1.0$ is marked "Airscrew" corresponding with the ordinary propeller. This portion of the curve approaches. an asymptate $x=$ I.I (approx.) in the direction of the negative axis and is marked in this portion of the curve as "The Intermediate Brake State." From. this abscissa onwards the ordinate decreases from $+\propto$ to a value of I.4 at $x=1.3$, when it increases gradually to a point at $x=2$ marked as "The Standing State." This branch of the curve is marked "Turbine." For small negative values of relative pitch the "Specific function" has two nearly equal negative values, represented by a loop with a cusp at the origin, and corresponding with the "Ist Brake State of Work." From near the end of the loop defining this part of the curve it is discontinuous from about $x=-0.3$ to -0.6 , and is then represented by a straight line to a point with co-ordinates $x=1.5, y=1.6$. (approx.), which corresponds to "The Standing State." This straight line is defined as the " and Brake State of Work" and the loop as "The Vortex Ring State of Work."

In the article considerable emphasis is laid on the phenomenon discovered by the author in a purely analytical manner. It is named "The Vortex Ring Working State," and is represented by the dotted discontinuous part of the curve. In explanation, he imagines the screw working in the state of brake, as is the case for a large negative relative pitch, and considers the progressive lessening of its translational speed. Under these conditions a moment arrives when a surface of discontinuity is formed in the wake of the screw across which the fluid will not flow. Directly after its formation the surface of discontinuity divides into two surfaces and a vortex ring, the axis of which coincides with the axis of the screw and appears in the space thus formed. The two surfaces of discontinuity which enclose the vortex ring move progressively apart, and a moment arrives when one of these surfaces crosses the space swept by the blades of the screw. The moment corresponds to the change of direction of the stream of fluid which flows across the plane of the screw, and at once the screw tends to make an infinite number of revolutions.

Referring to the positive portion of the curve, the "Specific function," after reaching a maximum, diminishes rapidly and, passing through nil value, comes to a short interval of breakage, which passes asymptotically to the turbine work of the screw. In this latter interval the "Specific function" represents the inverse of the efficiency of the turbo-motor. After passing a minimum which corresponds to the maximum of the turbo-motor efficiency, the "Specific function," by a parabolic branch, quasirectilinear, disappears into infinity, corresponding to the stoppage of the screw in a current which is directed on the screw. 
After reference to various authors on hydrodynamics and to the work done on discontinuous motion by Kutta and Karman, Professor Bothezat elahorates his own conception of dealing with fluid motion problems, which he calls he "Empirico-theoretic" method. In general, this may be represented as follows :"All the space in which a hydrodynamic phenomenon has played is divided into two sorts of regions. In one of these the hydrodynamic resistances are, so to say, concentrated, in the other they are absent. The hydrodynamic resistances once experimentally determined, the connection between the two sorts of regions is established by means of the general theorems of mechanics and hydrodynamics, the phenomena which take place in the second sort of regions being considered as under the laws of perfect fluids." This conception is applied to the theory of the screw, but not elaborated in any detail. ("Aviation," May 15, 1918.)

\section{WIND AT VARIOUS HEIGHTS.}

The results of pilot balloon observations made at the Zentral-Anstalt für Meteorologie und Geodynamik, Vienna, in the years I9II-I9I4 have been analysed by von Berger and A. F. La Roche. The total number of ascents dealt with is 996 , of which 284 exceeded $5 \mathrm{~km}$. and 79 exceeded $9 \frac{1}{2} \mathrm{~km}$. The data at the disposal of the authors were the mean velocities for $\frac{1}{2} \mathrm{~km}$. stages, published by the Zentral-Anstalt for each ascent. The stages or zones have been numbered from I. to XX.; the lowest stage numbered $\mathrm{I}$. is from ground level, $200 \mathrm{~m}$. to $500 \mathrm{~m}$. Each of the others covers $500 \mathrm{~m}$. The winds above $10 \mathrm{~km}$. are not discussed. The results are set out in tables and in diagrams. Some of the results are given below :-

(I) The average speed of the wind varies from $5.4 \mathrm{~m} / \mathrm{s}$ in stage $I$. and $7.8 \mathrm{~m} / \mathrm{s}$ in stage II. to $I I .8 \mathrm{~m} / \mathrm{s}$ in stage XX. Increase of wind speed in going from one stage to the next occurred in about two-thirds of the cases, the proportion being as high as 80 per cent. for the step from stage I. to stage II.

(2) The maximum wind-speed recorded at any height on any day during the month is given much prominence in the tables. Such a maximum usually occurs just before the balloon is lost to sight, a fact which should be borne in mind in the discussion. In nine months out of the 48 the maximum occurred in stage $V$. (though never in stage IV.). The favourite direction for the maximum was W.N.W., which is credited to ro months. The highest maximum, $39 \mathrm{~m} / \mathrm{s}$, occurred in September, 1912. The height above ground at which it was found is not stated.

(3) Taking all ascents together, the favourite direction for the wind in stage $I$. was W.; in stages II. to VIII., W.N.W. or N.W., and in stages IX. to XX., W.N.W. or W. On the average, winds within $90^{\circ}$ of W.N.W. are about four times as frequent as winds in the other two quadrants.

(4) In an investigation of the steadiness of the wind the following definitions are adopted:-

Gerader Kurs (Straight Course).-A change in direction of not more than $1 I^{\circ}$ i $5^{\prime}$ (presumably between one stage and the next). $90^{\circ}$. Biegung (Bending).-A change in a single quadrant, not more than

Wendung (Reversal).-A change between $90^{\circ}$ and $180^{\circ}$.

Schlinge (Loop).-A change of more than $180^{\circ}$.

The cases of "Schlinge" are presumably those indicated by footnotes in the original reports.

For stage II., Gerader Kurs is entered in 54 per cent. of cases, Biegung in 42 per cent., Wendung in 3 per cent., and Schlinge in I per cent. For stage X. the corresponding percentages are $70,28,2,0$, and for stage XX. 79, 19, I, I. (J. Van Berger and A. F. La Roche, "Osterreichische Flug-Zeitschrift," Feb. and Mar., 19I8.) 


\section{AERIAL WAVES AND WIND DIRECTION.}

On October 1, 1917, a disturbance, presumably due to an air wave, was reported at places near the coast of Holland. Doors flew open and shut, pictures swung on the walls, and windows clattered. It is stated that, according to the official report from the Meteorological Institute at de Bilt, the disturbance was to be attributed to an explosion which occurred at a munitions factory in the North of England. Accepting this explanation of the phenomenon, the author regards it as evidence of a strong current from N.W. in the upper air this current is identified with the one which carried the German airships over France when returning from a raid on England on October 20, and is referred to as the summer monsoon prolonged into the autumn. (W. Krebs, "Osterreichische FlugZeitschrift,"' Jan., I918.)

\section{BIBLIOGRAPHY.}

The New Giant German Aeroplane. ("The Engineer," June 14, 1918.)

Aluminium Piston for Aeroplane Engines. ("Engineering," July 5, 1918.)

The Gallaudet Airscrew Propulsion Described. ("Aviation and Aeronautical Engineering," May I, I918:)

The Sopwith Triplane. ("Flight," April 4, 1918. Illus.)

Aviation and the War. Discussion of the training of aviators; also some remarks on the classification of machines. (C. F. Lee, "Journal of the Washington Academy of Sciences," April 19, 19r8.)

The Effect of an Aeroplane Rudder in Flight. Describes methods of finding the moments about the centre of gravity of aeroplanes of different length due to rudders of different aspect ratios under varying angles of deviation. The results are expressed in the form of tables and curves. (Karl H. White, "Aviation and Aeronautical Engineering," May 1, 1918. 4 Figs.)

\section{RECENT ADDITIONS TO THE LIBRARY.}

"The Travel of Circular Depressions and Tornadoes and the Relation of Wind to Circular Isobars." By Sir Napier Shaw, F.R.S. London. I9I8. Meteorological Office. 4 to, 44 pp. Illustrated. Geophysical Memoirs No. I 2 .

"Painting by Immersion and by Compressed Air"" By A. S. Jennings. Illustrated. Oct., $27^{2}$ pp. London. 1918. E. and F. N. Spon. 3s. 6d.

"History of the Metallurgy of Iron and Steel." By Sir Robert Hadfield. London. I9ז5. Institute of Mechanical Engineers. Illus.

" Unsolved Problems in Metallurgy." By Sir Robert Hadfield. London. rgo5. Institute of Civil Engineers. Illus.

" History of Metallurgy." By Sir Robert Hadfield. London. I905. Iron and Steel Institute. Illus.

“Fuel." By Sir Robert Hadfield. London. 1918. Institute of Gas Engineers. Illus.

"Aeronautics." By A. E. Berriman. 19r8. National Home Reading Union. London. $3 \mathrm{~d}$. Oct. pp. 24.

"Aeronautics in Theory and Experiment." By W. L. Cowley and H. Levy. 1918. London. Edward Arnold. Oct. Illus. pp. 278 . i6s.

" Outwitting the Hun." By Pat O'Brien. 1918. London. Wm. Heinemann. Oct. pp. $265.6 \mathrm{~s}$. 